\title{
Isolated A1 Pulley Rupture of Left Fourth Finger in Kendo Players: Two Case Reports
}

\author{
Jin Hyung Lee, $\mathrm{MD}^{1}$, Hyoung Seop Kim, $\mathrm{MD}^{2}$, Seung Ho Joo, $\mathrm{MD}^{3}$
}

\begin{abstract}
${ }^{1}$ Department of Rehabilitation Medicine, Yonsei University College of Medicine, Seoul; Departments of ${ }^{2} \mathrm{Physical}$ Medicine and Rehabilitation, ${ }^{3}$ Diagnostic Radiology, National Health Insurance Service Ilsan Hospital, Goyang, Korea
\end{abstract}

Annular pulley injury of fingers is usually observed in rock climbers who support their entire body weight with flexed fingers during climbing. But these lesions can also follow trivial trauma, such as lifting heavy objects with the fingertips, or during sports and recreational activities. The A2 and A4 pulleys are most usually involved and reported most frequently. However, traumatic Al pulley rupture has not been reported yet, to the best of our knowledge. Kendo is a very vigorous martial art with frequent physical contact. Therefore, we reported two cases of repetitive microtraumatic left fourth finger Al pulley rupture in Kendo players with results from physical examination and imaging studies, such as ultrasonography and magnetic resonance imaging, together with related literature.

Keywords Athletic injuries, Finger injuries, Ultrasonography

\section{INTRODUCTION}

A series of retinacular structures around the synovial tendon sheath of the fingers thicken the sheath at 5 specific points, forming the annular pulley system (pulleys A1-A5); while additional fibers that crisscross between the annular pulleys create the cruciate pulley system (pulleys C1-C3). These pulleys combine to prevent excursion of the flexor tendons from the metacarpophalangeal and interphalangeal joints during finger flexion

Received July 7, 2014; Accepted November 17, 2014

Corresponding author: Hyoung Seop Kim

Department of Physical Medicine and Rehabilitation, National Health Insurance Service Ilsan Hospital, 100 Ilsan-ro, Ilsandong-gu, Goyang 10444, Korea

Tel: +82-31-900-0137, Fax: +82-31-900-0343, E-mail: iskrakhs@gmail.com

(c) This is an open-access article distributed under the terms of the Creative Commons Attribution Non-Commercial License (http://creativecommons. org/licenses/by-nc/4.0) which permits unrestricted noncommercial use, distribution, and reproduction in any medium, provided the original work is properly cited.

Copyright $\odot 2015$ by Korean Academy of Rehabilitation Medicine
[1]. Therefore, annular pulley injuries lower mechanical efficiency and reduce digital performance.

Annular pulley ruptures may occur during powerful flexion of fingers with the metacarpophalangeal joint in extension and proximal interphalangeal joint in flexion. Pulley ruptures usually involve long fingers, particularly the A2 pulley of the fourth finger of the non-dominant hand [2]. Since these injuries are typically detected in rock climbers, they are commonly referred to as climber's finger [3]. In general, A2 and A4 pulleys are most commonly involved, especially the A2 pulley tear. The pathomechanics of closed annular pulley rupture is initial partial tear of the distal end of A2 pulley followed by progression to complete rupture and involvement of the A3 and A4 pulleys. However, the A1 pulley is rarely involved [2].

Isolated Al pulley rupture has not been reported yet. Thus, we reported two cases of Kendo players with isolated Al pulley rupture of the left fourth finger, together 
with related literature.

\section{CASE REPORTS}

\section{Case 1}

A 24-year-old national Kendo athlete visited the outpatient Department of Physical Medicine and Rehabilitation for left fourth and fifth finger pain ongoing for a year. The patient was diagnosed with 'trigger finger' at a local orthopedic clinic and other oriental traditional medicine clinics. The patient was treated with local injection and acupuncture several times without improvement of symptoms.

The patient received Kendo training for 4 hours a day since elementary school and used protective gloves most of the time. The patient was usually pain free, but despite the absence of definite trauma history, there was aggravation of pain at the left fourth and fifth metacarpal bone area during training.

There was no difference between both hands at resting state, but the left fourth finger tendon protruded from the palm when fingers were flexed against resistant force (Fig. 1). On manual muscle test examination, there was mild left side weakness of Medical Research Council (MRC) good grade of the fourth and fifth finger flexors, but no

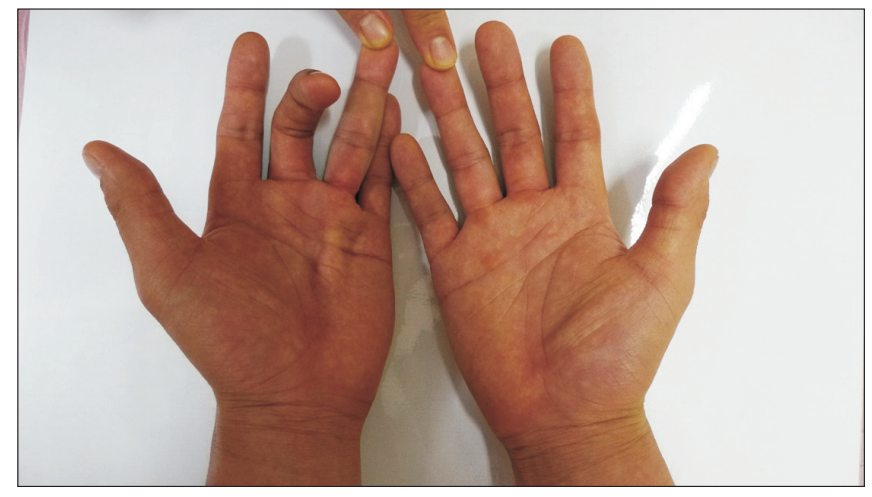

Fig. 1. Physical examination of both hands during finger motions. There is no difference between both palms during finger extension, but the protrusion of left fourth finger flexor tendon from palm is demonstrated during resistive finger flexion, as compared to the right side.
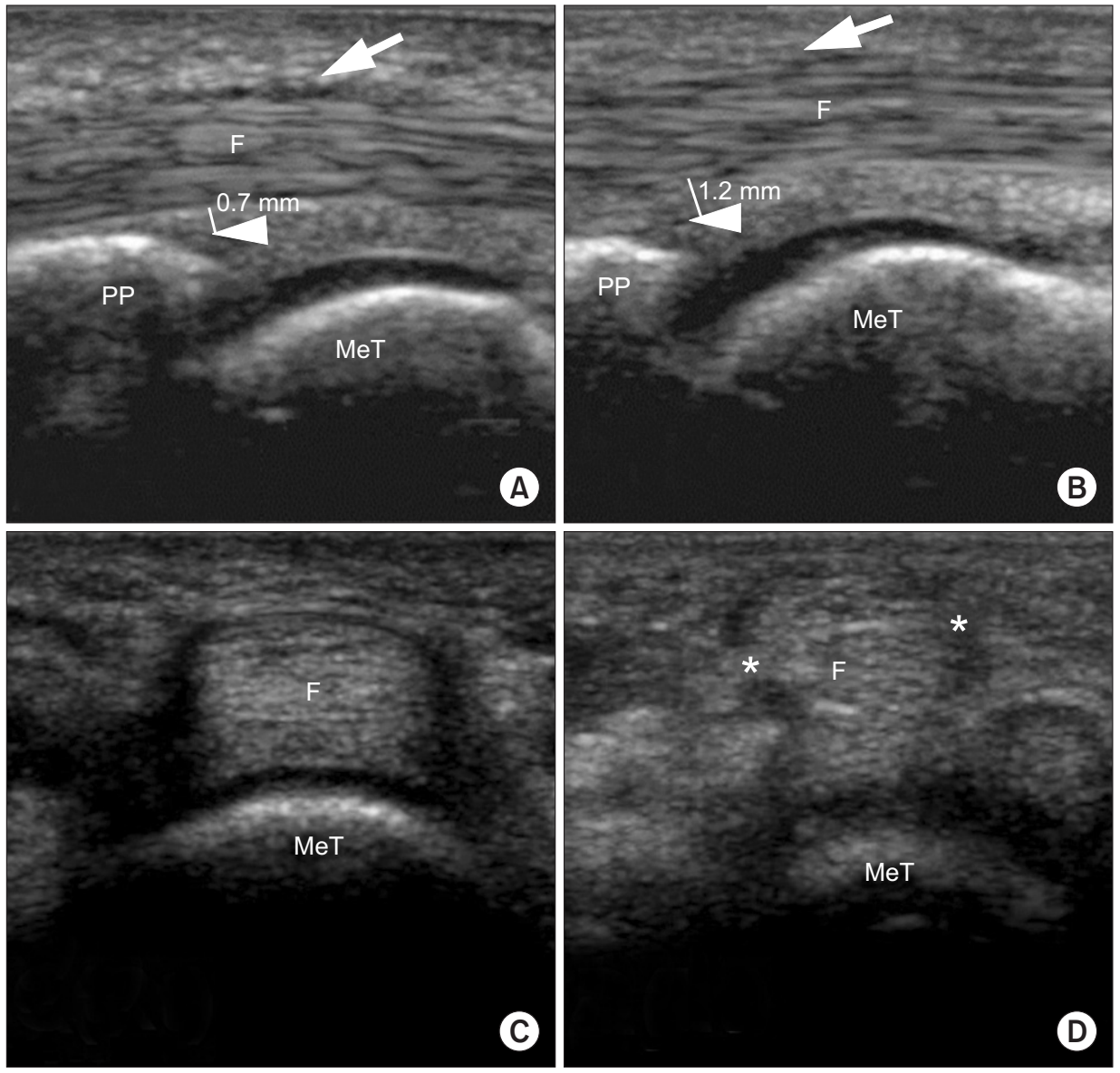

Fig. 2. Ultrasonography of first patient's fourth fingers at metacarpophalangeal joint area during active forced flexion position. The Al pulley (arrow) is observed in right side (A), but it (arrow) is not observed in left side (B) and flexor tendon is separated from volar plate (arrow head) (B). Left fourth Al pulley is not observed and the flexor tendon is in contact (asterisks) with subcutaneous tissue directly (D), as compared to right fourth Al pulley (C). PP, proximal phalanx; MeT, metacarpal head; F, flexor tendon. 
abnormality when they were extended or abducted.

On sensory nerve testing no abnormality on pinprick, touch and vibration stimulation was observed. Electromyography evaluation to rule out possible ulnar nerve injury showed results within normal limits. The patient was referred to a radiologist to evaluate for finger flexor tendon lesions.

A radiologist with at least 3 years of musculoskeletal ultrasonographic experience performed the ultrasonographic examinations using a 5-12 MHz iU22 (Philips Medical System, Bothell, WA, USA) linear array transducer. Ultrasonographic evaluation was performed on the extended fingers in resting position from the head of the metacarpal bones to the distal phalanges on supine position. Active forced flexion against resistance was provided by the radiologist with his fingertips to ensure the highest tendon detachment.

The Al pulley of the left fourth finger was not observed and the flexor tendon was detached from the volar plate in the longitudinal scan on ultrasonography (Fig. 2). Furthermore, the difference of tendon detachment between rest and forced finger flexion was unclear. However, the tendon was detached about $1.2 \mathrm{~mm}$, as compared to the contralateral side. Magnetic resonance imaging (MRI) was performed to confirm the lesion at the patient's finger, which showed signal changes at the flexor tendon that was considered as tendinosis on sagittal scan view. Ultimately, the left fourth flexor tendon was detached from the metacarpal head on axial scan (Fig. 3).

\section{Case 2}

A 53-year-old amateur Kendo athlete visited the outpatient Department of Physical Medicine and Rehabilitation complaining of left fourth metacarpophalangeal joint pain. He had received Kendo training for 20 years and used protective gloves most of the time. Like the above case, the patient did not feel pain during rest, but pain was present at the left fourth and fifth metacarpal area during Kendo training. Similarly, the left fourth finger tendon protruded clearly from the palm when counter force was applied to both fourth fingers.

A physiatrist with 5 years of musculoskeletal ultrasonographic experience performed the ultrasonographic examinations using a 5-12 MHz Voluson i (GE Healthcare, London, UK) linear array transducer with the same method as in the first case.
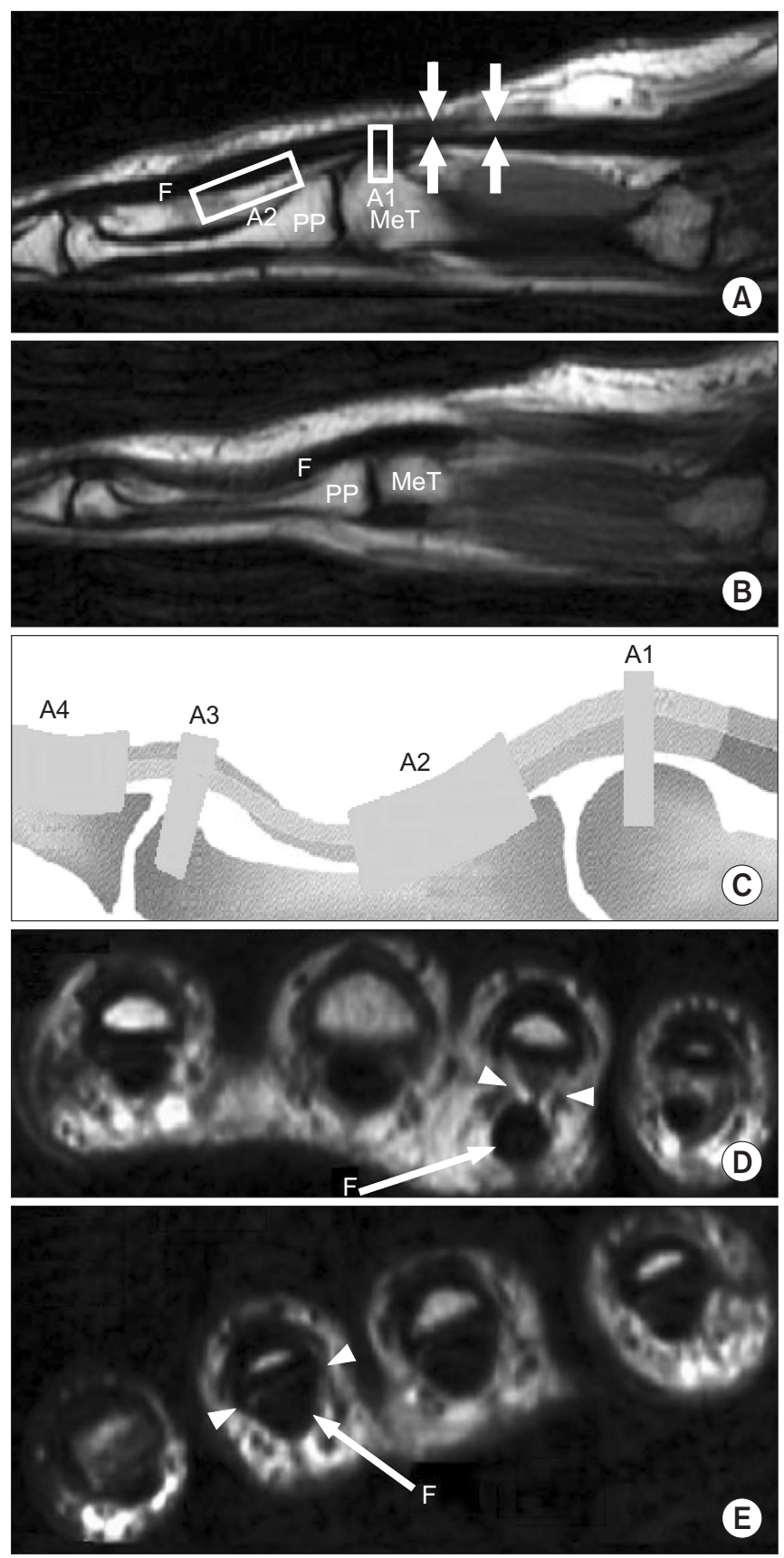

Fig. 3. Proton density MR images of both fourth finger flexor tendons. The flexor tendon of left fourth finger (A) is not anchored to metacarpal head and detached (arrow), as compared to flexor tendon of right fourth finger (B). A2 pulley is located at proximal region of proximal finger (C), which is intact in left finger (A). Axial MR images also show Al pulley injury (arrowhead) on left fourth finger (D), as compared to right fourth finger (E) [9]. PP, proximal phalanx; MeT, metacarpal head; F, flexor tendon; MR, magnetic resonance. 

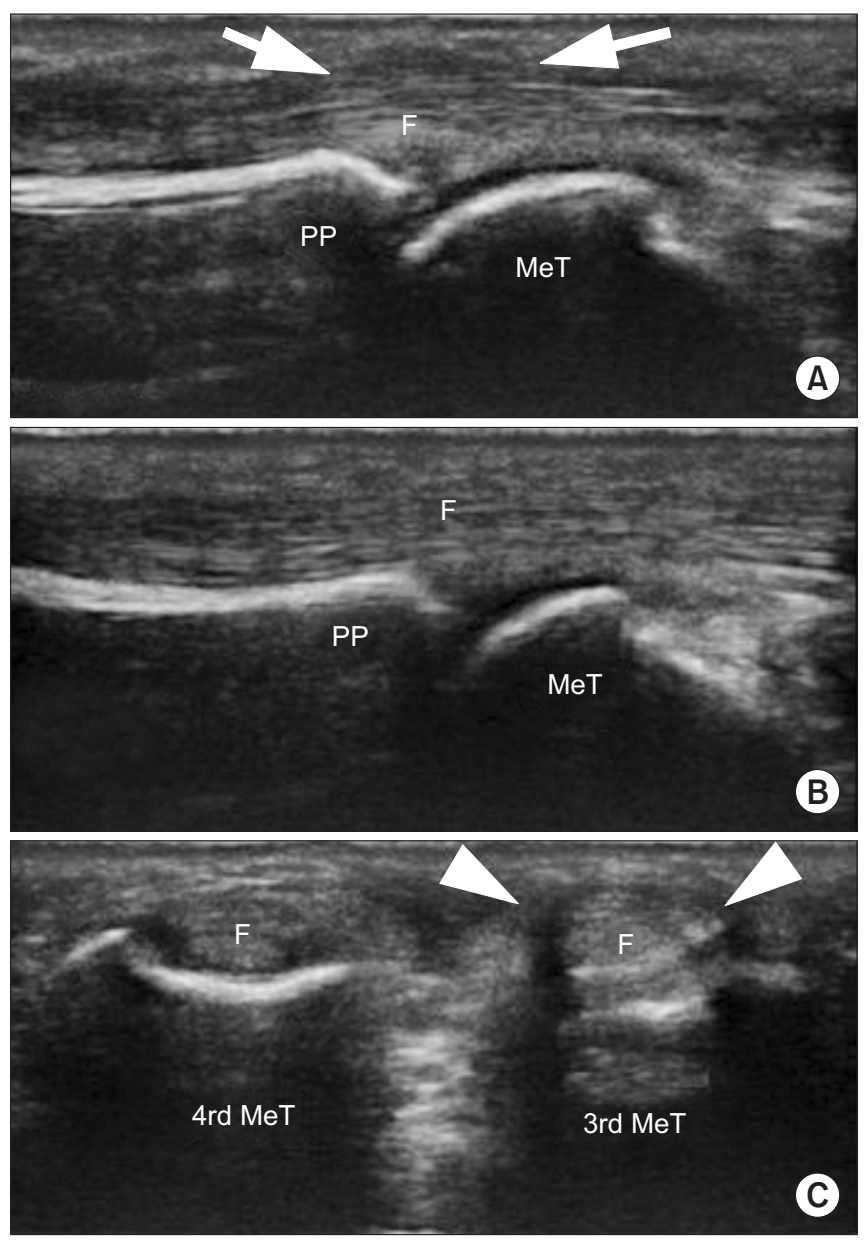

Fig. 4. Ultrasonographic findings of the second patient's fourth fingers at metacarpophalangeal joint area (bilateral) during active forced flexion position. The Al pulley (arrow) is observed at the right side (A), but not observed at the left side (B), and the 3rd Al pulley (arrow head) is observed (C). However, the left fourth A1 pulley is not observed and the flexor tendon is in contact with subcutaneous tissue directly (C). PP, proximal phalanx; MeT, metacarpal head; F, flexor tendon.

Detachment of the flexor tendon from the volar plate was not clear on longitudinal scanning, but the hypoechoic Al pulley was not observed in longitudinal and transverse views (Fig. 4). Though MRI was not performed due to refusal by the patient, we were able to diagnose isolated Al pulley rupture with thorough history taking and ultrasonographic evaluation.

\section{DISCUSSION}

Kendo, the Japanese sword art, is a popular martial art in Korea and is a vigorous exercise with frequent physical contact but low injury risk with proper use of safety equipment [4].

When a Kendo player grasps a bamboo sword, the right hand is placed in front of the left hand. A player uses the right hand as a fulcrum point and the left hand as the main source of force. Thus, a Kendo player grasps the bamboo sword more forcefully with the left hand than the right hand, and the distal end of the bamboo sword is placed in the mid palm of the left hand for smooth changes in direction during swinging of the sword. The two patients in our case also followed this method. Since protective gloves are worn at all times, wrist and finger injuries have been rarely reported during Kendo sparring. Injuries in Kendo occur more commonly at the foot, ankle, heel, glenohumeral joint, shoulder joint and knee joint, as compared to other martial arts $[4,5]$.

Pulley system injuries mainly involve the A2 to A4, while A1 and A5 pulley injuries are rarely reported $[2,6]$. However, the cause of isolated Al pulley rupture in Kendo players is considered to be the grasping method of the bamboo sword (Fig. 5). When sparring with an opponent, most of the impact force is concentrated at the left fourth metacarpophalangeal joint because the distal end of bamboo sword is located around the fourth metacarpophalangeal joint, and such chronic microtrauma is thought to cause Al pulley rupture.

When A2 pulley ruptures occur, pain aggravates with finger flexion, and maximal volar displacement occurs over the proximal phalanx [6]. However in our cases, physical examination showed that volar displacement occurred over the palm and not over the proximal phalanx when counter force was applied on the left fourth fingertip.

A dynamic study with active forced flexion of an involved finger is usually required to detect A2 pulley injuries with ultrasonography. With the probe placed on the affected finger with active forced flexion against resistance, the presence of pathological increment of distance between the flexor tendon and phalanx (tendon-phalanx distance) in longitudinal plane is considered as pulley injuries [6,7]. Klauser et al. [6] reported that the tendonphalanx distance was greater with active forced flexion than at rest; in addition, the tendon-phalanx distance of complete injury was greater than that of incomplete injury with active forced flexion. They also noted that the 

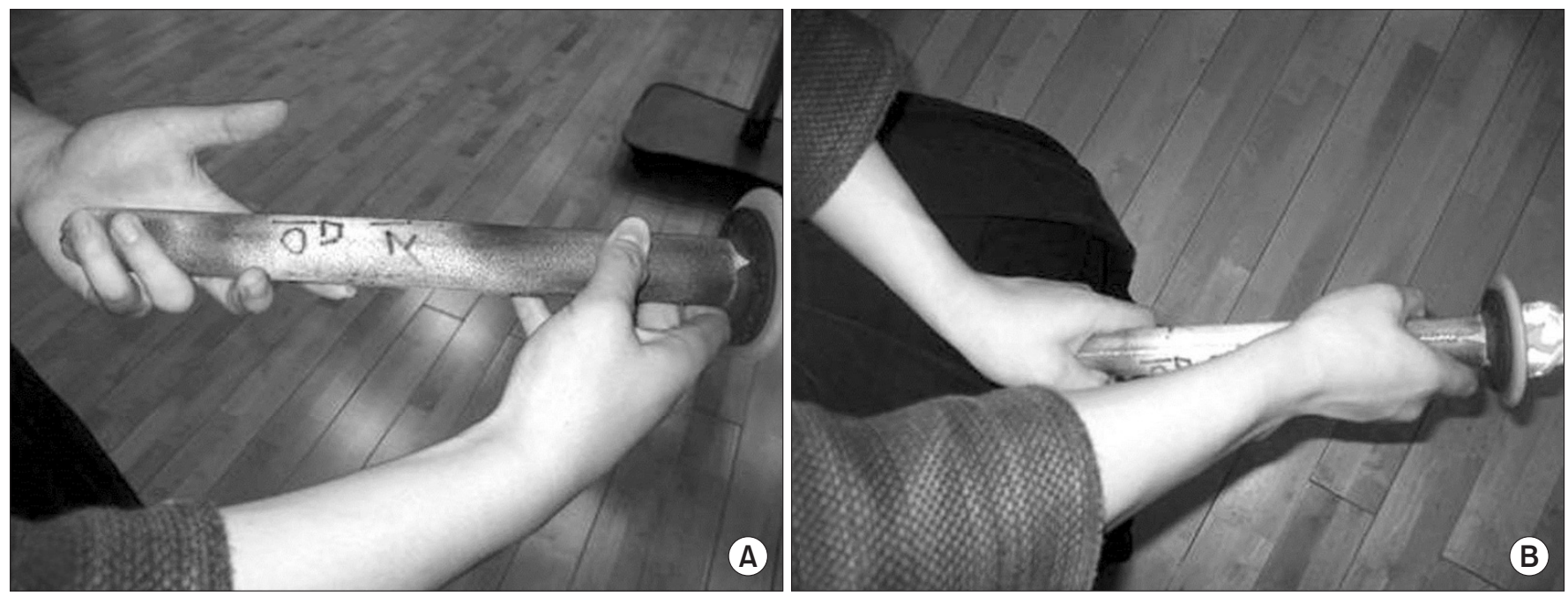

Fig. 5. Bamboo sword grip method. The Kendo player usually grips the bamboo sword anteriorly with his/her right hand and posteriorly with his/her left hand. (A, B) The tip of the bamboo sword is located at the left palm.

observed distance increased with the number of pulleys involved. Based on their study and previous reports, a distance of $\geq 1.0 \mathrm{~mm}$ is indicative of pulley system injury and $<3.0 \mathrm{~mm}$ with forced flexion is considered a sign of incomplete rupture of the A2 pulley.

However, in our cases, scanning with active forced flexion did not show considerable increment of tendonphalanx distance, as compared with scanning on rest. This difference was possibly due to the anatomical site of the pulley i.e., the A2 pulley is located at the mid-shaft of proximal phalanx between the metacarpophalangeal joint and the proximal interphalangeal joint, causing easy displacement of the specific finger flexor tendon from the phalanx on injury. However, since the Al pulley is located just above the metacarpophalangeal joint, the probe must be placed in the middle of the joint to detect Al pulley injuries. Thus, in Al pulley injuries, ultrasonographic examination with active forced flexion may not show a difference in increment of tendon-phalanx distance compared with the ultrasonographic examination at rest, as in A2 pulley injuries.

Transverse views in ultrasonography did not provide any additional significant information in the evaluation of A2 pulley injury [6]. However, on transverse view in the second case, hypoechoic rims that represent the left fourth Al pulley were not observed and fourth finger flexor tendon was closer to the subcutaneous tissue, as compared to its neighboring or contralateral tendon. It can also be explained by anatomical position. As the Al pulley is located at the mid palm area, transverse view evaluations with ultrasonography reveals Al pulley injuries more clearly than other pulley injuries. Thus the transverse view study is as important as the longitudinal view study in detecting Al pulley injuries.

MR images also provide additional assessment of annular pulley injuries [7]. Sagittal and axial MR images directly depict tears as a defect of the pulley. However, MR imaging is more time-consuming, more costly and less universally available, as compared with ultrasonography [8]. Furthermore, MR imaging, does not enable real-time evaluations such as active forced flexion. For detection of pulley injuries, ultrasonography demonstrated high accuracy, sensitivity, specificity, positive predictive value, and negative predictive value, as compared to MR imaging. In the second case, we were able to diagnose Al pulley injury with careful physical examination and ultrasonography alone, without taking MR images.

In conclusion, two cases of isolated Al pulley rupture were diagnosed in Kendo players. The probable cause of the injury was the grasping method in which the left hand grasps the cylindrical handle firmly and the distal tip of bamboo sword is located around the mid left fourth metacarpal bone. This causes the impact force to be transferred to the Al pulley when making swinging, slicing and stabbing motions with the sword during sparring. These were the first diagnosed cases of isolated Al pulley rupture in Kendo players based on history and physical examination, ultrasonography and MR images. 


\section{CONFLICT OF INTEREST}

No potential conflict of interest relevant to this article was reported.

\section{REFERENCES}

1. Lee JC, Healy JC. Normal sonographic anatomy of the wrist and hand. Radiographics 2005;25:1577-90.

2. Marco RA, Sharkey NA, Smith TS, Zissimos AG. Pathomechanics of closed rupture of the flexor tendon pulleys in rock climbers. J Bone Joint Surg Am 1998; 80:1012-9.

3. Bollen SR. Injury to the A2 pulley in rock climbers. J Hand Surg Br 1990;15:268-70.

4. Jeong HJ, Kim DS, Sim YJ, Choi SB. Musculoskeletal pain in young Kendo player. J Korean Acad Rehabil Med 2008;32:553-7.
5. Song HS, Park SJ, Han SK, Nah KH, Cheung HK, Choi WK, et al. Analysis of sports injuries in Kendo. J Korean Orthop Sports Med 2005;4:122-7.

6. Klauser A, Frauscher F, Bodner G, Halpern EJ, Schocke MF, Springer P, et al. Finger pulley injuries in extreme rock climbers: depiction with dynamic US. Radiology 2002;222:755-61.

7. Hauger O, Chung CB, Lektrakul N, Botte MJ, Trudell D, Boutin RD, et al. Pulley system in the fingers: normal anatomy and simulated lesions in cadavers at MR imaging, CT, and US with and without contrast material distention of the tendon sheath. Radiology 2000;217: 201-12.

8. Stoller DW. Magnetic resonance imaging in orthopaedics and sports medicine. Baltimore: Lippincott Williams \& Wilkins; 2007.

9. Bianchi S, Martinoli C. Ultrasound of the musculoskeletal system. Berlin: Springer; 2007. 